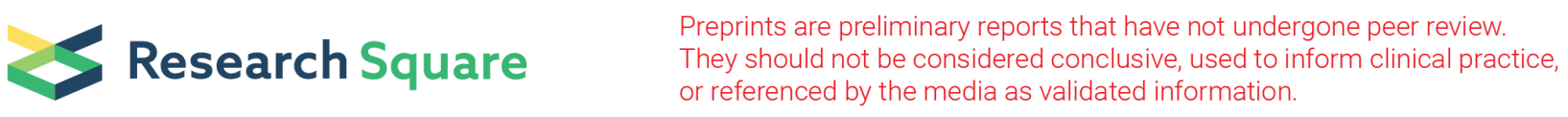

\title{
Improved COVID-19 detection using data augmentation Deep Convolution GAN and classifier DenseNet.
}

Debmitra Ghosh ( $\sim$ debmitra.ghosh@gmail.com )

JIS University https://orcid.org/0000-0002-1887-7631

\section{Research Article}

Keywords: Deep learning, convolutional neural networks, generative adversarial networks, synthetic data augmentation, COVID-19 detection

Posted Date: February 22nd, 2021

DOl: https://doi.org/10.21203/rs.3.rs-235624/v1

License: (c) (i) This work is licensed under a Creative Commons Attribution 4.0 International License.

Read Full License 


\title{
Improved COVID-19 detection using Deep Convolution GAN and DenseNet.
}

\author{
Debmitra Ghosh \\ M.Tech \\ JISIASR \\ debmitra.ghosh@gmail.com
}

\begin{abstract}
SARS-CoV-2 or severe acute respiratory syndrome coronavirus 2 is considered to be the cause of Coronavirus (COVID-19) which is a viral disease. The rapid spread of COVID-19 is having a detrimental effect on the global economy and health. A chest X-ray of infected patients can be considered as a crucial step in the battle against COVID-19. On retrospections, it is found that abnormalities exist in chest X-rays of patients suggestive of COVID-19. This sparked the introduction of a variety of deep learning systems and studies which have shown that the accuracy of COVID-19 patient detection through the use of chest X-rays is strongly optimistic. Although there are certain shortcomings like deep learning networks like convolutional neural networks (CNNs) need a substantial amount of training data but the outbreak is recent, so it is large datasets of radiographic images of the COVID-19 infected patients are not available in such a short time. Here, in this research, we present a method to generate synthetic chest X-ray (CXR) images by developing a Deep Convolution Generative Adversarial Network-based model. In addition, we demonstrate that the synthetic images produced from DCGAN can be utilized to enhance the performance of CNN for COVID-19 detection. Classification using CNN alone yielded 85\% accuracy. Although there are several models available, we chose MobileNet as it is a lightweight deep neural network, with fewer parameters and higher classification accuracy. Here we are using a deep neural network-based model to diagnose COVID-19 infected patients through radiological imaging of 5,859 Chest X-Ray images. We are using a Deep Convolutional Neural Network and a pre-trained model "DenseNet 121" for two new label classes (COVID-19 and Normal). To improve the classification accuracy, in our work we have further reduced the number of network parameters by introducing dense blocks that are proposed in DenseNets into MobileNet. By adding synthetic images produced by DCGAN, the accuracy increased to 97\%. Our goal is to use this method to speed up COVID-19 detection and lead to more robust systems of radiology.
\end{abstract}

Keyword- Deep learning, convolutional neural networks, generative adversarial networks, synthetic data augmentation, COVID-19 detection.

\section{INTRODUCTION}

By the end of the year 2019 in Wuhan, a respiratory disease caused by severe acute respiratory syndrome coronavirus 2 (SARS-CoV-2) was named Coronavirus disease. COVID-19 was initially detected in Wuhan, China, in December 2019, and has spread worldwide since then leading to the ongoing 2020 coronavirus pandemic. COVID-19 positive cases are increasing daily with more than 4.18 million cases and 286,000 deaths have been registered in more than 200 countries and territories as of 12 May 2020. Since no vaccines or cures exist, the only efficient way of human protection against COVID-19 is to reduce spread by prompt testing of the population and isolation of the infected individuals. Certain health symptoms combined with a chest X-ray can be used to diagnose this infection. A chest X-ray can be used as a visual indicator of coronavirus infection by radiologists. The learning models and tests have shown that it is highly likely that patients with COVID-19 infection are detected correctly by using chest radiography images. The world is fighting a battle now; this war is all about CORONA VIRUS. We came to know about this not-so-familiar virus last year December, when news of people getting sick due to some unknown reason broke news channels from Wuhan, China. The outbreak which started in 2019 and caused by Corona Virus is called COVID-19. Corona means "crown" in Latin, the structure of this virus shows some has a spike that looks like a crown, hence the name derived. COVID-19 is coined from the term Corona Virus Disease and 19 because it was first identified in December 2019. On 11th February WHO officially declared this disease, the International Committee on Taxonomy of Viruses has named it as severe acute respiratory syndrome coronavirus 2", or SARS-CoV-2, it was previously referred to as 2019, novel Corona Virus or 2019-nCoV by WHO. Patients with COVID-19 interpreted from Chest X-Ray images can guide to the four stages, i.e., (a) early stage (0-4 days): GGO should be present, (b) progressive stage (5-8 days): GGO will increase largely with crazy paving which is identified in the image by patchy marks (like stone patches marks), (c) peak stage (9-13 days): Consolidation which is identified as lung 
tissue filled with liquid, (d) absorption stage ( $>14$ days): With improvement, fibrous stripes. Every disorder can be characterized by the specific nature of the tissue which can be examined through existing modalities. Ground Glass Opacity (GGO) is identified in the early stages of X-Rays soon fibrous stripes appear and resolution occurs in the lungs. World Health Organization has declared Corona Virus as pandemic on 11th March 2020. In India, 12759 people are infected with the coronavirus till 16th April, according to the Ministry of Health \& Family Welfare (MoHFW) with 420 deaths. The diagnosis of COVID-19 is mainly performed from a swab taken from the patient's Nasopharynges. Collecting specimens from the upper and lower respiratory tracts and testing for swab is recommended by the CDC (Centre for Disease Control) Testing. CDC testing is mainly performed in the USA. In vitro testing also includes specimens or blood taken from a suspect's body which included phlebotomists to draw blood from one's finger. Specimens are required to be stored at specific temperatures with proper protection and also shipped for testing to the laboratory from clinics. Hence test result gets delayed with increased quarantined time.

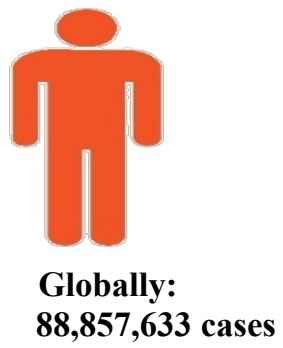

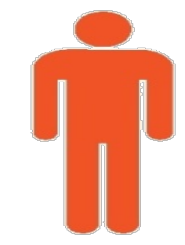

India $10,430,330$ cases

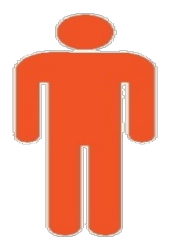

Globally:

Death 1,913,336

Figure: Corona Virus records as available. This data is collected on 08-01-2021 from worldometers (https://www.worldometers.info/coronavirus)

Problems faced with these diagnostic strategies:

- Specimens are required to be stored at $2-8^{\circ} \mathrm{C}$ and also shipped for testing. Infrastructure is required to store specimens

- Testing-kits are already less available in India. The cost of an RT-PCR kit is about USD 120-130.

- RT-PCR test can take up to 2 days to complete. Hence test result are delayed with increased quarantined time.

- Phlebotomist is required for the testing purpose they are getting exposed to invasive swab.

- Prone to human-error and bias Cost-effective approach.

- Special biosafety lab is required to store a PCR machine which each cost about USD 15,000 to USD 90,000. Testing-kits are less available in the market. The cost of an RT-PCR kit is about USD 120-130.

- Time-consuming approach.

- Infrastructure is required to store specimens. A special biosafety lab is required to store a PCR machine which each cost about USD 15,000 to USD 90,000.

Existing solutions are:

- Nucleic Acid Testing

- Protein Testing

- $\quad$ Pool Testing

Other methods of diagnosis include clinical approach, positive radiological image (Computed Tomography or Chest X-Ray), positive pathogenic tests, etc. This project aims at detecting COVID-19 at an early stage of infection. The current scenario across the globe is heartbreaking, thousands of people are affected, in this context, various researchers have contributed a lot in measuring lung health and facilitating diagnostic inference through the expert's opinion. Despite the fact, the traditional probing mechanisms involve Radiologists but this disease is highly infectious so a computer-aided system can help in reducing the chances of transmitting the disease from patients to health workers. Here we are using data collected from the people of Italy and China. Our image database includes infected, died, and new cases of COVID-19. We need more data to train our model for better accuracy. We are primarily focusing on developing Artificial Intelligence-based software which will be operational on radiological images to identify COVID-19 and other lung diseases. To facilitate these our deep learning-based computational model for characterization of Lung conditions in health and pathology using Chest 
$\mathrm{X}$-Ray images is designed. Convolutional neural networks (CNNs) have attained state-of-the-art performance in the field of medical imaging, given enough data [1]-[4]. Such performance is accomplished by training on labeled data and tuning its millions of parameters. CNN can easily over on small datasets because of the large number of parameters, therefore, the efficiency of generalization is proportional to the size of the labeled data. The small datasets of medical imaging domain are the biggest challenge [5]-[7]. The medical image dataset is also of limited quantity and variety of samples. The medical image collection is a very expensive and tedious process that requires the participation of radiologists and researchers [6]. The outbreak of COVID-19 occurred recently so a sufficient dataset of infected person positive chest X-ray (CXR) images is difficult to gather. We propose to alleviate the drawbacks by using synthetic data augmentation.

\section{BACKGROUND}

\section{A. Data Augmentation}

To extend the training dataset artificially, Data augmentation methods are employed. Current data augmentation techniques use simple modifications to incorporate affinity like image transformations and color adjustments, such as scaling, converting, improving contrast or brightness, blurring, and sharpening, white balance, etc. [8]. This classical data augmentation is fast, reliable, and easy. However, here we have a slightly altered sample, the changes are limited because it is structured to turn an existing sample whereas, classical data augmentation does not produce completely unseen data. To overcomes the limitations of classical data augmentation a modern, advanced form of augmentation is Generative Adversarial Network (GAN) which is one such innovative model that generates synthetic images. GAN generates unseen samples with a min-max game without supervision [9]. The method GAN follows is to use two opposing networks: $\mathrm{G}(\mathrm{z})(\mathrm{G}(\mathrm{z})$ generator) and $\mathrm{D}(\mathrm{z})(\mathrm{D}(\mathrm{z})$ discriminator), where the generator aims to produce a realistic image to trick the discriminator that is well trained to better differentiate between the real and fake images. The aim of the generator is to minimize the cost value function $\mathrm{V}$ $(D, G)$ whereas the discriminator maximizes it [10].

\section{B. Classification}

To reduce human interpretation computer image classification is used which helps to analyze and classify images into certain categories. It is an important and growing field of computer vision. Because the features are very important to classification, most of the researches on image classification focuses on image feature extraction and classification algorithms. SIFT and HOG are traditional image features that involve manually designed. So, it triggered automatically features extraction methods by using the prior knowledge of the known categories and helped to avoid the traditional image classification methods which was a complicated process of feature extraction. The usage of Convolutional neural networks was supported for its ability to automatically learn, adapt, and organize at the same time, extracting features expressively and efficiently.

Deep convolutional neural network (CNN) has been successfully used in the field of computer vision, such as image classification [11], target tracking [12], target detection [13], and semantic image segmentation [14, 15]. Krizhevsky et al. presented an AlexNet [11] model, in the ImageNet Large Scale Visual Recognition Challenge 2012 (ILSVRC2012), which has about 60 million parameters and eight layers. VGG [16] has 16-layer, GoogleNet [17] have also achieved great success with Inception as the basic structure, and ResNet [18] with residual blocks that can alleviate the problem of gradient disappearance. The deep CNN model is itself a dense computational model, the drawbacks of this model are a large number of parameters, huge computing load, and large memory access lead to heavy power consumption, which makes it difficult to apply the model to devices with limited hardware resources. To apply the deep CNN model to different real-time applications it is required to have a lowmemory portable, it is compressed and accelerated to reduce parameters, computation cost, and power consumption. Denil et al. [19] proved that the parameters of the deep convolutional neural networks have a lot of redundancy, and these redundant parameters have little influence on the classification accuracy. Denton et al. [20] found an appropriate low-rank matrix to estimate the information parameters of deep CNNs by singular value decompositions. High computational cost and more retraining to achieve convergence is required for this method. Han et al. [21] deleted the unimportant connections in the pre-trained network by parameter pruning, retrained and quantized the remaining parameters. To reduce the compression rate and quantized parameters Hoffman coding is used. However, the method requires manual adjustment of super parameters. Chen et al. [22] used a low-cost Hash function to group the weights between the two adjacent layers into a Hash bucket for weight sharing, which reduces the storage of additional positions and realizes parameter sharing. Hinton et al. [23] compressed the network model by knowledge distillation and extracted useful information. The simple network and the complex network have similar performance as the useful information is migrated to a smaller and simpler network. In addition, many related types of research have improved network models to compress networks. For 
example, SqueezeNet [24] is a network model based on fire module, MobileNets [25] is a network model based on depthwise separable filters, and ShuffleNet [26] is improved on the basis of the residual structure by introducing group pointwise convolution and channel shuffle operation. To reduce parameters and computation MobileNet uses depthwise separable convolution (unlike the VGG-16 network) to deepen the network. At the same time, the classification accuracy of MobileNet on the ImageNet data set only reduces by $1 \%$. However, in order to be better applied to mobile devices with limited memory, the parameters and computational complexity of the MobileNet model need to be further reduced, so dense blocks are used as the basic unit in the network layer of MobileNet. This network sets a small growth rate, and has fewer parameters and lower computational cost. The ultimate goal of this new models, Dense-MobileNets, is to achieve high classification accuracy.

MobileNet: MobileNet is a streamlined architecture that uses depthwise separable convolutions to construct lightweight deep convolutional neural networks and provides an efficient model for mobile and embedded vision applications [25]. The structure of MobileNet is based on filters which are separable convolution filters of

Figure: Layers of DenseNet 121 network.

depthwise convolution filters and point convolution filters. The point convolution filter combines the output of depthwise convolution linearly with $1 * 1$ convolutions and depthwise convolution filter performs a single convolution on each input channel. Diagrammatic representation of MobileNet is given below.

Dense Connection: DenseNet [27] proposed a new connection mode, connecting each current layer of the network with the previous network layers so that the input features of previous layer is taken and passed to current layer the output feature maps of all the previous layers this helps to alleviate the problem of gradient disappearance. Since each layer is connected with all the previous layers, the previous features can be repeatedly used to generate more feature maps with less convolution kernel. The basic unit modules of DenseNet is dense blocks. With a growth rate of 4 , each dense block structure consists of 4 densely connected layers. Each layer in this structure takes the output feature maps of the previous layers as the input feature maps. Different from the residual unit in ResNet [18], which combines the sum of the feature maps of the previous layers in one layer, the dense block transfers the feature maps adding the dimension of the feature maps to all the subsequent layers. The dense block superimposes the number of feature maps of the previous convolution layers so, only the magnitude is required to be equal. The hyperparameter growth rate is used by DenseNet to control the number of feature map channels in the network. The growth rate indicates that the output feature maps of each network layer are. That is, for each convolution layer, the input feature maps of the next layer will increase channels. Diagrammatic representation of DenseNet is given below.

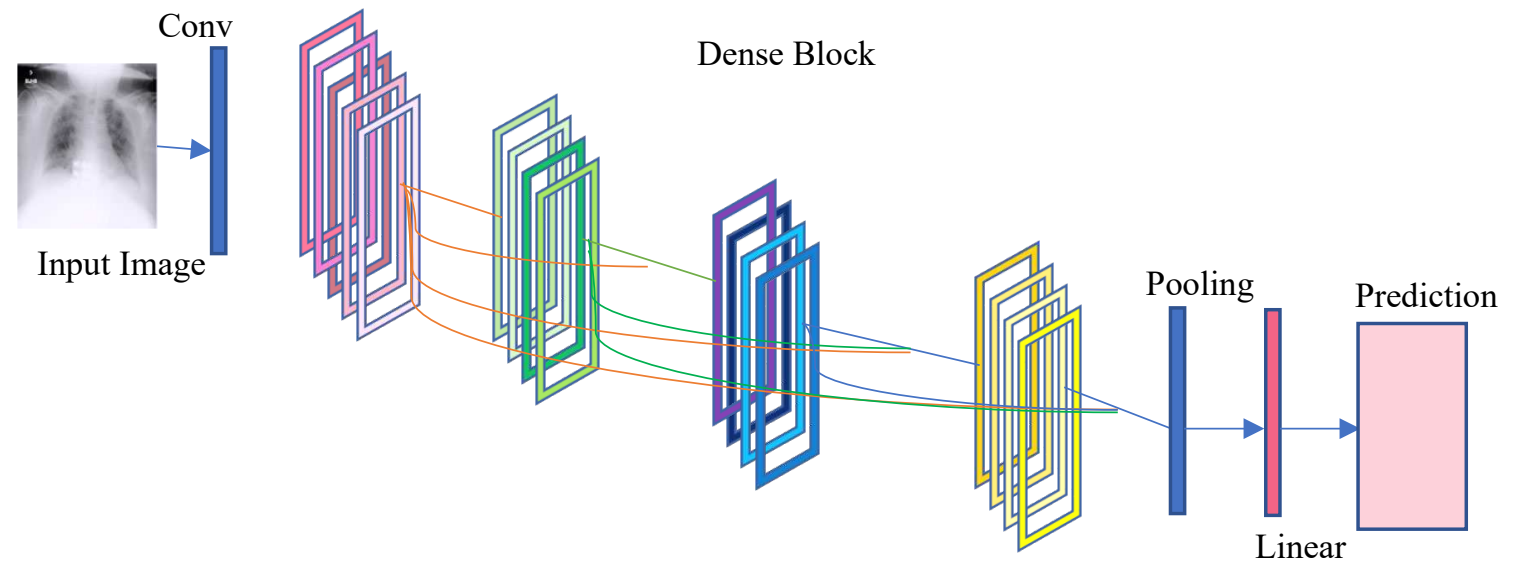

Figure: DenseNet Architecture.

CNN Architecture and layers: Convolutional Neural Network or CNN is widely used in image processing because of its feature engineering character. It mainly consists of three layers. These are convolutional layer, pooling layer, and completely connected layer. These can be divided into two blocks hidden block and classifier block. The first block consists of a convolutional layer with an activation function. This block acts for feature 
map. Diagrammatic representation of Deep Convolution Neural Network is given below.

Then the second layer is a classification which consists of fully connected and SoftMax layers. CNN provides many advantages:

- $\quad \mathrm{CNN}$ is used in medical imaging for feature extraction of images having low resolution.

- Another important task performed by CNN is image classification.

- Segmentation is also done by CNN. It is being used for object detection.

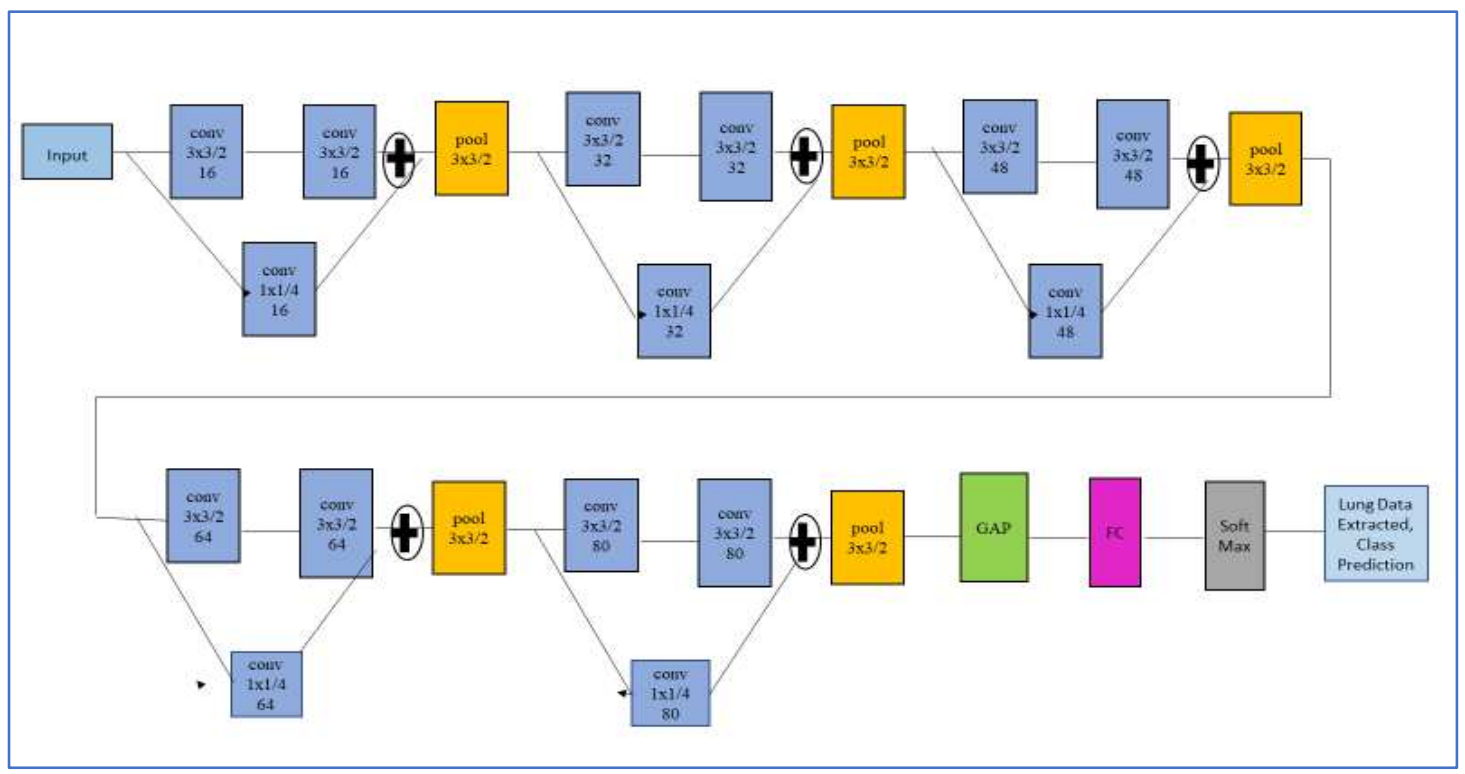

Figure: Shows 2D CNN architecture.

\section{LITERATURE SURVEY}

After the outbreak of novel coronavirus pandemic in 2020 scientist and researcher across the world is trying to find drugs, medicine, and vaccines of this disease. Research on every field virology, sensor aided detector, protein-based analysis etc are being carried out. Computer science researchers are using AI-aided model for early diagnosis by using image processing techniques in Chest X-Ray images to find a solution. Radiologists from China and The United States came together to identify COVID-19 (219) cases from general viral pneumonia (205) cases by examining Radiological images (424). It was found that early detection of this disease can be done from radiological images with high accuracy. AI-based approach:

1. Li et at [28] used a 3D convolutional Res-Net 50 model which takes 4,356 input as Chest CT images from 3,322 patients. These images are collected from 6 Chinese Hospitals. Max pooling operation is done to combine features extracted from CT images and finally fully connected layer to computer probability of 3 classes i.e. non-pneumonia, community accrued pneumonia, and COVID-19. 1,296 images of COVID-19, 1,735 for community accrued pneumonia and 1,325 non-pneumonia patients. The class which shows the highest probability will be the predominant class. The area under the receiver operating characteristics curve value is obtained at 0.96

2. Xu et al [29] used a CNN model using Res-Net 18 which takes input as pulmonary CT images. Here 3 classes are predicted using the trained model i.e. 224 images of influenza-A viral pneumonia, 175 images of healthy lungs, and 219 from 110 COVID-19 patients. The class which shows the highest probability will be the predominant class. The accuracy of this model is $86 \%$.

3. Ghosal et al [30] used a Bayesian CNN model using a drop-weight approach which takes input as 5,941 posterior-anterior chest radiological images. Here 4 classes are predicted using the trained model i.e. 2,786 images of bacterial pneumonia, 1,504 non-COVID-19 viral pneumonia,1,583 images of healthy lungs, and 68 COVID-19 patients. The class which shows the highest probability will be the predominant class. The accuracy of this model is $89.92 \%$. 
4. Wang et al [31] used a modified inception transfer-learning model which takes input as 1,065 CT images. Here 2 classes are predicted using the trained model i.e. 740 viral pneumonia and 325Covid-19 patients. The class which shows the highest probability will be the predominant class. The accuracy of this model is $79.3 \%$ with a specificity of 0.83 and a sensitivity of 0.67

5. Bai et al [32] used a multi-layer perceptron with Long short-term memory (LSTM) model which takes input as $133 \mathrm{CT}$ images of critical patients. The area under the receiver operating characteristics curve value is obtained at 0.954 .

6. Jin et al [33] used a 2D deep Convolutional Neural Network model which takes input as $970 \mathrm{CT}$ images. Here 2 classes are predicted using the trained model i.e. 1,385 negative cases and 496 COVID19 patients. The class which shows the highest probability will be the predominant class. The accuracy of this model is $94.98 \%$ with AUC of $97.91 \%$.

7. Jin s et al [34] used a combination of 3D UNet++ and ResNet50 model which takes input as 1,136 CT images. Here 2 classes are predicted using the trained model 723 COVID-19 patients. The class which shows the highest probability will be the predominant class. Accuracy and sensitivity are 0.974 and specificity of 0.922 .

8. Narin et al [35] used a pre-trained ResNet50 model which takes input as $100 \mathrm{CT}$ images. Here 2 classes are predicted using the trained model i.e. 50 normal cases and 50 COVIA-19 patients. The class which shows the highest probability will be the predominant class. The accuracy of this model is $98 \%$.

9. Wang et al [36] used a Deep Convolutional Neural Network model namely COVID-NET which takes input as 16,756 chest radiological images. Here the trained model shows 13,645 COVID-19 patients. The class which shows the highest probability will be the predominant class. The accuracy of this model is $92.4 \%$.

10. Chowdhury et al [37] used a combination of AlexNet, DenseNet-201, SqeezeNet 201, and ResNet18 model which takes input as Chest X-ray images. Here 3 classes are predicted using the trained model 190 COVIA-19 patients, 1,341 normal patients, and 1,345 viral pneumonia. The class which shows the highest probability will be the predominant class. Accuracy $98.3 \%$ on X-Ray images.

11. Maghdid et al [38] used a combination of pre-trained AlexNet and CNN model which takes input as 170 Chest XRay images and 361 CT images. Accuracy $98 \%$ on X-Ray images and $94.1 \%$ on CT images.

12. Zhao et al. [39] developed a model to generate synthetic images for lung-nodules classification multiscale using VGG16 network and a DCGAN based model, Forward and Backward GAN (F\&BGAN).

13. Beers et al. [40] trained a PGGAN (progressively grown generative adversarial network) to generate augmented medical images of fundus pictures to show premature retinopathic vascular pathology (ROP) and glioma multimodal MRI pictures.

14. Dai et al. [41] used a GAN model to produce segmented images of lung and heart from CXR.

15. Nie et al. [42] developed a patch-based GAN and an automated image optimization model to convert brain CT pictures to the MRI.

16. Xue et al. [43] used two models called a Segment and a Critic which are two GAN networks to study the connection between a binary brain tumor segmentation map and brain MRI pictures.

17. Schlegl et al. [44] used GAN to study anomaly detection in retinal images on patches of both unseen and healthy imagery the data distribution of healthy tissue using patches in the retinal region.

18. Wang et al in his research published in the journal Radiology [45], chest radiography outperformed laboratory testing in the detection of 2019 novel coronavirus disease. The frequency of anomalies in radiographical images rapidly increased after the onset of symptoms and peaked during the days of illness. 
19. Huang et al., and other researchers [46]-[47] in concluded that chest radiography should be used as the main COVID-19 screening method (also known as SARS-CoV-2. Since the pandemic is recent, there are only a limited number of CXR images available for study. The generation of artificial data is very effective in the case of small datasets and when the data includes sensitive information.

20. Wang et al and other researchers [48]-[50]. in many research projects and innovations related to COVID-19 has been proposed.

\section{Methodology}

\section{A. Our Contribution}

Dense-MobileNet: Dense-MobileNet takes two-point convolutional layers and a depthwise convolutional layer which is basically a depthwise separable convolution as a whole, called a dense. The accumulated output feature maps generated by point convolutions in all previous depthwise separable convolution layers is the input feature maps of depthwise separable convolution layer. The input feature map in the point convolution layer is the output feature map generated by the depthwise convolution in the dense block. So, the dense block structure in this model only has one dense connection, In the Dense-MobileNet model, only one input feature map needs to overlay the output feature map. The Dense-MobileNet model does not add other transition layers too therefore after superimposing the output feature maps generated by the previous separable convolutions, the size of the feature map gets reduced by the depthwise convolution with stride 2. Finally, global average pooling is applied and connected directly to the output layer of the MobileNet model. Experiments show that the classification accuracy of the global average precooling depthwise separable convolution with dense connection before the global average pooling is higher than that of two-layer depthwise separable convolution without dense connection and finally the depthwise separable convolution layer before global average pooling is also densely connected.

In this study, we use CNN for COVID-19 detection. We combine synthetic CXR images generated using DCGAN with our proposed $\mathrm{CNN}$ architecture.

This research has the following contributions:

1) Propose a Deep Convolution Generative Adversarial Network (DCGAN) based GAN, for the generation of synthetic CXR images.

2) Design a CNN-based model for COVID-19 detection.

3) Using DCGAN for augmentation of the training dataset with our model for improved detection of COVID-19.

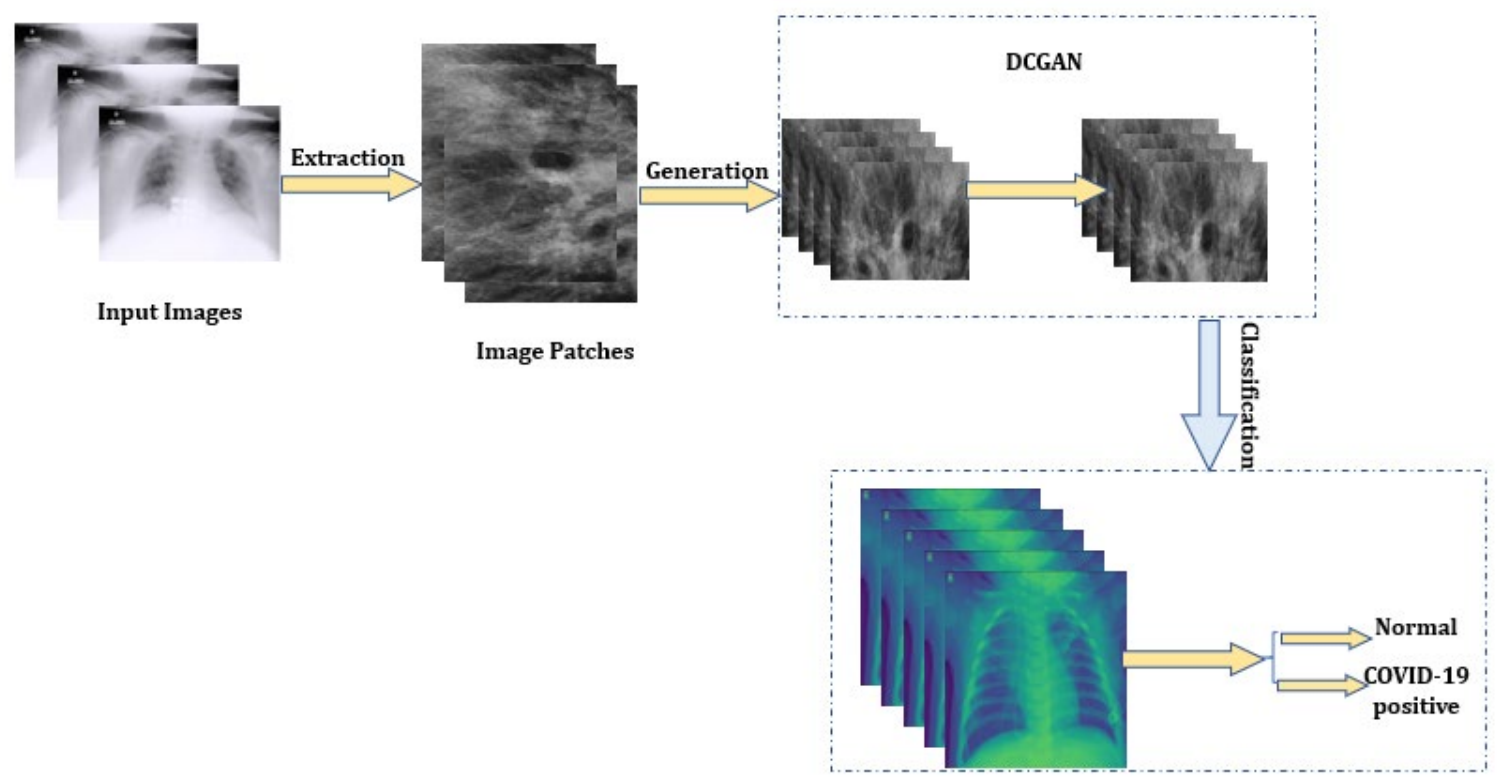

\section{B. Dataset generation}

The dataset is composed of 1124 CXR images., collected from images from three publicly accessible datasets: 1) IEEE Covid Chest X-ray dataset [51] 
2) COVID-19 Radiography Database [52] and

3) COVID-19 Chest X-ray Dataset [53]

- 403 images of COVID-CXR

- 721 images of Normal-CXR.

Sample of dataset are given below:

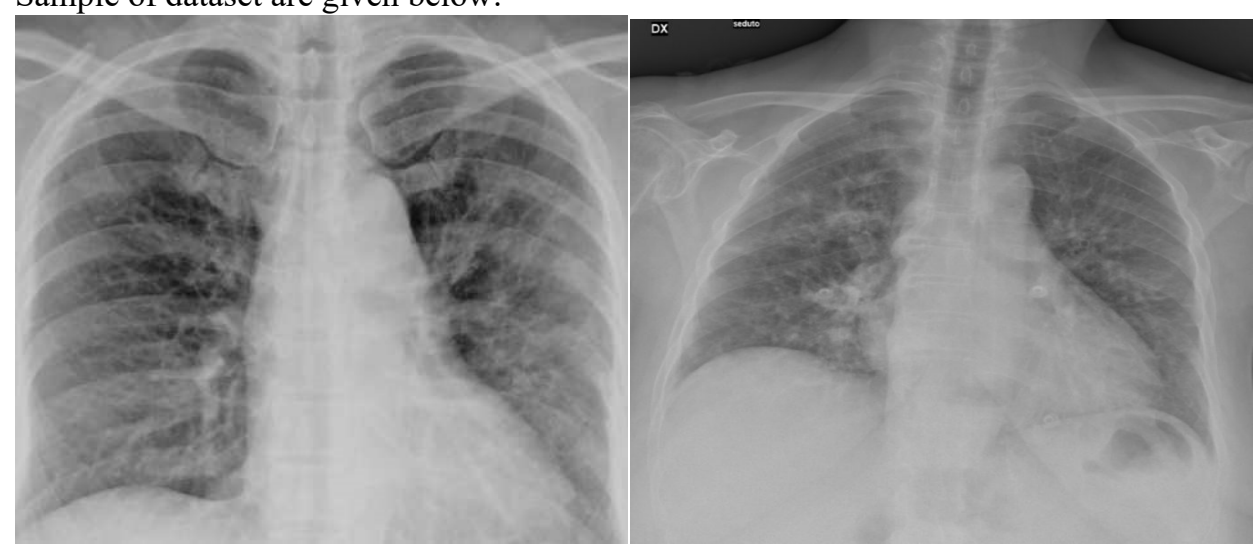

Figure: Representative Chest X-Ray image of COVID-19 patients

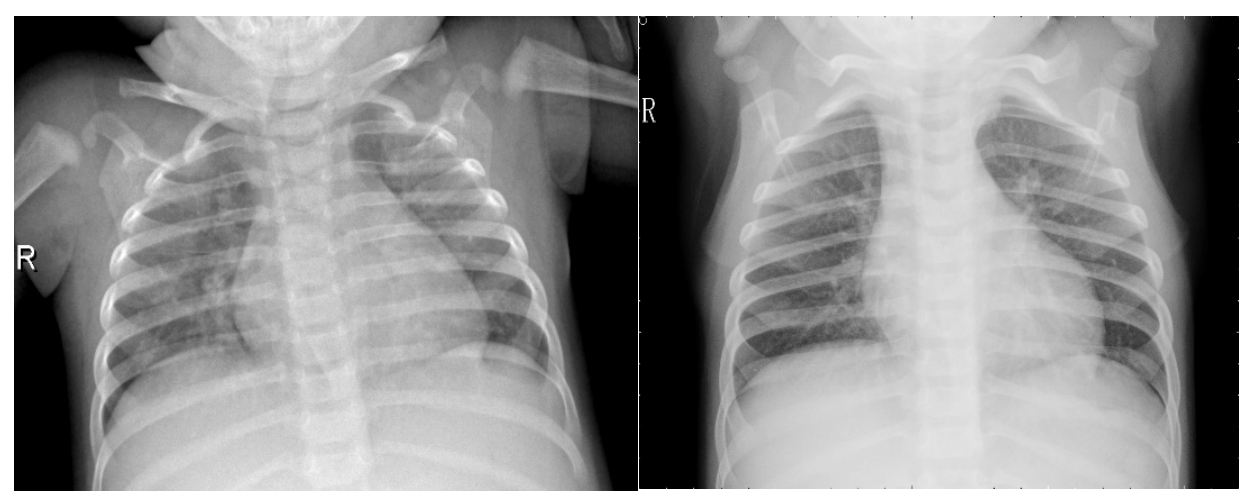

Figure: Representative Chest X-Ray image of Normal patients

\section{Classification}

The decision to develop the dataset on these three datasets is driven by the fact that all of them are opensourced and completely available to the public and research communities. All three datasets are merged together and duplicate images are removed. The hashing method is used to remove the duplicate images. This method creates a hash value that uniquely identifies an input. The most striking trend is the limited number of cases and scarcity of availability of Chest X-Ray images associated with COVID-19 patient's data in the public domain. The dataset consists of 932 training samples with COVID-19 Chest X-Ray images: 331 and Normal-Chest XRay images: 601 and 192 testing samples with COVID-19 Chest X-Ray images: 72 and Normal-Chest X-Ray images: 120 . The image preprocessing steps involved are resizing (112 X 112 X 3$)$ and each image is normalized by rescaling the pixels from $[0,255]$ to $[0,1]$. Adaptive Moment Estimation called Adam (Adam is a method for stochastic optimization which calculates adaptive learning rates for parameters.) [54] is used as the optimizer and categorical_cross_entropy as the loss function. The activation function is ReLU. The learning rates of Adam are controlled by the parameter beta (beta $=0.9$ ). The hyperparameters used for training are learning rate $=$ 0.001 , and batch_size $=16$. The network is trained for 25 epochs and after training, $85.4 \%$ accuracy is achieved. The proposed architecture is trained and tested using Keras deep learning library. 


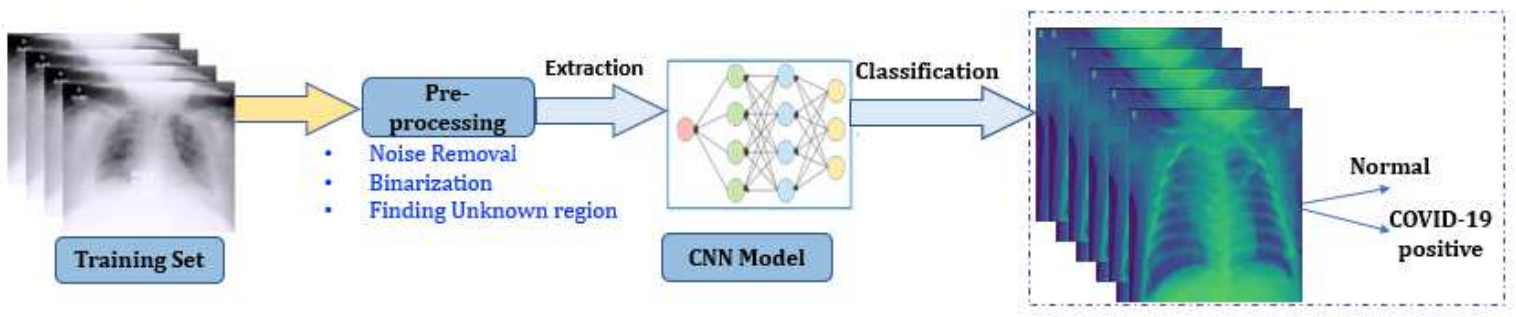

Figure: We enhanced image quality using image processing approach. The network includes first part for feature extraction and other part for binary classification of Covid-19 and normal.

\section{Generating Synthetic Data}

Generative Adversarial Networks (GANs) utilizes two neural networks that compete with one another to create new virtual instances of data that can be transmitted as real data [10]. GANs are extensively used for image generation. GAN to perform data augmentation. GANs generate high-resolution samples from highly variable data sets. The discriminator is provided with an image as well as a class label and it decides if the image is true or false. DCGAN applies the associated class label $\mathrm{c}$ and noise $\mathrm{z}$ to each produced sample [55]. The discriminator D gives a distribution of probability over class labels and sources.

$\mathrm{P}(\mathrm{S}$ I X); P(C I X) = D(X):

The log-likelihood of source class Ls and correct class Lc forms the objective function.

$\mathrm{Lc}=\mathrm{E}[\log \mathrm{P}(\mathrm{C}=\mathrm{c}$ I Xreal $)]+\mathrm{E}[\log \mathrm{P}(\mathrm{C}=\mathrm{c}$ I Xfake $)](1)$

$\mathrm{Ls}=\mathrm{E}[\log \mathrm{P}(\mathrm{S}=$ real I Xreal $)]+\mathrm{E}[\log \mathrm{P}(\mathrm{S}=$ fake I Xfake $)](2)$

D maximizes Ls + Lc and G maximizes Lc - Ls. We propose a GAN architecture based on DCGAN, to produces images which helped the classification network to get trained properly.

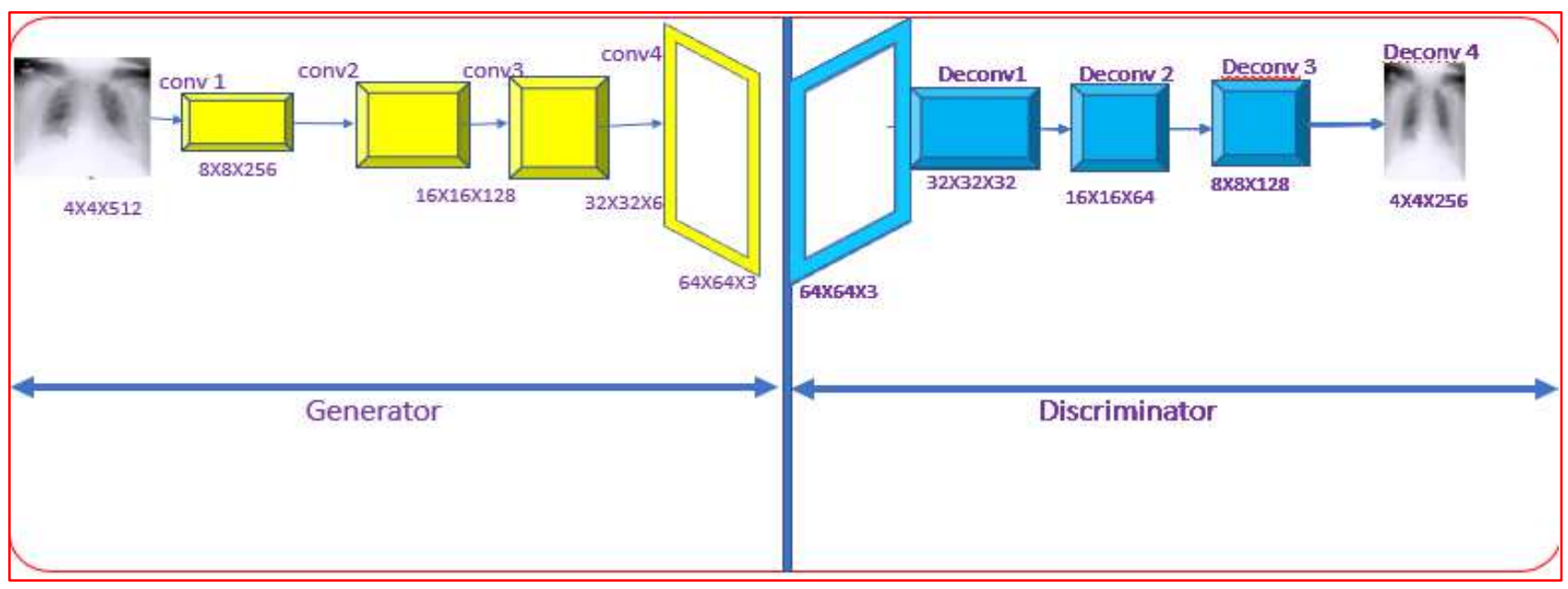

Figure: Diagrammatic representation of Deep Convolution Generative Adversarial Network. 


\section{RESULTS AND DISCUSSION}

In this section, we analyze the effect of the data augmentation technique used for COVID-19 detection. The Dense-MobileNet architecture was used initially, to perform COVID-19 detection. Then to improve the performance we used the synthetic data augmentation technique. The performance of the model is observed COVID-Chest X-Ray: 72 images and Normal-Chest X-Ray: 120 images on the testing, samples. We found that synthetic data augments produced enhanced the performance of CNN. An accuracy of $85 \%$ is achieved with actual data that increased to $97.4 \%$ with synthetic augments.

The implementation of the architecture is done using Keras [56] deep learning library in Colab.

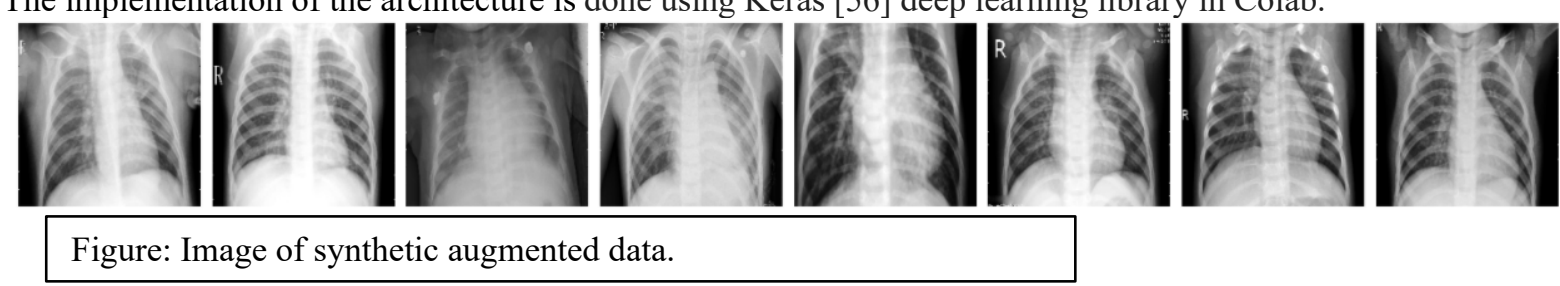

\section{CONCLUSION}

* The proposed CNN architecture classifies the two classes: - COVID-CXR and Normal-CXR.

* DCGAN helps to generate augmented data. Synthetic data augmentation enlarges the dataset.

- An improvement in classification performance from $85 \%$ to $97 \%$ accuracy is recorded when CNN is trained on actual data and synthetic augments.

* In future the quality of the synthetic CXR images can be improved by training different GAN model.

a. Limitation of Study:

- Architecture can be improved further based on more available database.

- The dataset is obtained from various sources and cross-center validations were not conducted in this analysis. We have made every effort to ensure that the data collected is correctly labeled. Any mistake in data labeling, however, would probably affect the results reported.

- It was found on retrospective review that Chest $\mathrm{X}$ ray has a really low sensitivity in detecting lung opacities but in advanced cases, it can definitely help. So now, the protocol is to have CXR + HRCT thorax (non contrast) for all patients. The major advantage is that it helps in picking up changes in the basal and peripheral portion of the lung as well as GGO.

- The only way to reliably detect COVID-19 is through medical assistance and clinical testing. However, this paper provides promising results that encourage the use of this approach to make more robust radiology systems. 


\section{REFERENCES}

[1] S. Wang, J. Sun, I. Mehmood, C. Pan, Y. Chen, and Y. Zhang, "Cerebral micro-bleeding identification based on a ninelayer convolutional neural network with stochastic pooling," Concurrency Comput., Pract. Exper., vol. 32, no. 1, p. e5130, Jan. 2020 .

[2] S. Wang, C. Tang, J. Sun, and Y. Zhang, "Cerebral micro-bleeding detection based on densely connected neural network," Frontiers Neurosci., vol. 13, p. 422, 2019.

[3] C. Kang, X. Yu, S.-H.Wang, D. Guttery, H. Pandey, Y. Tian, and Y. Zhang, “A heuristic neural network structure relying on fuzzy logic for images scoring," IEEE Trans. Fuzzy Syst., early access, Jan. 13, 2020, doi: 10.1109/TFUZZ.2020.2966163.

[4] G. Litjens, T. Kooi, B. E. Bejnordi, A. A. A. Setio, F. Ciompi, M. Ghafoorian, J. A. W. M. van der Laak, B. van Ginneken, and C. I. Sánchez, “'A survey on deep learning in medical image analysis," Med. Image Anal., vol. 42, pp. 6088, Dec. 2017.

[5] H. R. Roth, L. Lu, J. Liu, J. Yao, A. Seff, K. Cherry, L. Kim, and R. M. Summers, "Improving computer-aided detection using convolutional neural networks and random view aggregation," IEEE Trans. Med. Imag., vol. 35, no. 5, pp. 11701181, May 2016, doi: 10.1109/ tmi.2015.2482920.

[6] H. Greenspan, B. van Ginneken, and R. M. Summers, "Guest editorial deep learning in medical imaging: Overview and future promise of an exciting new technique," IEEE Trans. Med. Imag., vol. 35, no. 5, pp. 11531159, May 2016, doi: 10.1109/tmi.2016.2553401.

[7] N. Tajbakhsh, J. Y. Shin, S. R. Gurudu, R. T. Hurst, C. B. Kendall, M. B. Gotway, and J. Liang, “Convolutional neural networks for medical image analysis: Full training or ne tuning?" IEEE Trans. Med. Imag., vol. 35, no. 5, pp. 12991312, May 2016, doi: 10.1109/tmi.2016.2535302.

[8] A. Mikolajczyk and M. Grochowski, "Data augmentation for improving deep learning in image classication problem," in Proc. Int. Interdiscipl. PhD Workshop (IIPhDW), May 2018, pp. 117122.

[9] L. Engstrom, D. Tsipras, L. Schmidt, and A. Madry, "A rotation and a translation sufce: Fooling CNN with simple transformations," 2017, arXiv:1712.02779. https://arxiv.org/abs/1712.02779

[10] I. Goodfellow, J. Pouget-Abadie, M. Mirza, B. Xu, D. Warde-Farley, S. Ozair, A. Courville, and Y. Bengio, "Generative adversarial nets," in Proc. Adv. Neural Inf. Process. Syst., 2014, pp. 26722680.

[11] A. Krizhevsky, I. Sutskever, and G. E. Hinton, "Imagenet classification with deep convolutional neural networks," in Advances in Neural Information Processing Systems, vol. 25, pp. 1097-1105, MIT Press, Cambridge, MA, USA, 2012.View at: Google Scholar

[12] N. Wang and D. Y. Yeung, "Learning a deep compact image representation for visual tracking," in Advances in Neural Information Processing Systems, pp. 809-817, MIT Press, Cambridge, MA, USA, 2013.View at: Google Scholar

[13] W. Wang, C. Tang, X. Wang, Y. Luo, Y. Hu, and J. Li, "Image object recognition via deep feature-based adaptive joint sparse representation," Computational Intelligence and Neuroscience, vol. 2019, Article ID 8258275, 9 pages, 2019.View at: Publisher Site $\mid \underline{\text { Google Scholar }}$

[14] W. Wang, Y. Yang, X. Wang, W. Wang, and J. Li, "The development of convolution neural network and its application in image classification: a survey,” Optical Engineering, vol. 58, no. 4, Article ID 040901, 2019.View at: Publisher Site | Google Scholar

[15] F. Li, C. Wang, X. Liu, Y. Peng, and S. Jin, "A composite model of wound segmentation based on traditional methods and deep neural networks," Computational Intelligence and Neuroscience, vol. 2018, Article ID 4967290, 1 page, 2018.View at: $\underline{\text { Publisher Site }} \mid \underline{\text { Google }}$ $\underline{\text { Scholar }}$

[16] K. Simonyan and A. Zisserman, "Very deep convolutional networks for large-scale image recognition," in Proceedings of the International Conference on Learning Representations, San Diego, CA, USA, May 2015.View at: Google Scholar

[17] C. Szegedy, W. Liu, Y. Jia et al., "Going deeper with convolutions," in Proceedings of the IEEE Conference on Computer Vision and Pattern Recognition, pp. 1-9, Boston, MA, USA, June 2015.View at: Publisher Site | Google Scholar

[18] K. He, X. Zhang, S. Ren, and J. Sun, "Deep residual learning for image recognition," in Proceedings of the IEEE Conference on Computer Vision and Pattern Recognition, pp. 770-778, Las Vegas, NV, USA, June 2016.View at: Publisher Site | Google Scholar

[19] M. Denil, B. Shakibi, L. Dinh, M. A. Ranzato, and N. De Freitas, "Predicting parameters in deep learning," in Proceedings of the Advances in Neural Information Processing Systems, pp. 2148-2156, Lake Tahoe, NV, USA, December 2013.View at: Google Scholar

[20] E. L. Denton, W. Zaremba, J. Bruna, Y. LeCun, and R. Fergus, "Exploiting linear structure within convolutional networks for efficient evaluation," in Advances in Neural Information Processing Systems, pp. 1269-1277, MIT Press, Cambridge, MA, USA, 2014.View at: Google Scholar

[21] S. Han, H. Mao, and W. J. Dally, "Deep compression: compressing deep neural networks with pruning, trained quantization and huffman coding," 2015, https://arxiv.org/abs/1510.00149. View at: Google Scholar

[22] W. Chen, J. Wilson, S. Tyree, K. Weinberger, and Y. Chen, "Compressing neural networks with the hashing trick," in Proceedings of the International Conference on Machine Learning, pp. 2285-2294, Lille, France, July 2015.View at: Google Scholar

[23] G. Hinton, O. Vinyals, and J. Dean, "Distilling the knowledge in a neural network," 2015, https://arxiv.org/abs/1503.02531.View at: Google Scholar

[24] F. N. Iandola, S. Han, M. W. Moskewicz, K. Ashraf, W. J. Dally, and K. Keutzer, "SqueezeNet: AlexNet-level accuracy with 50x fewer parameters and $<0.5$ MB model size," 2016, https://arxiv.org/abs/1602.07360.View at: Google Scholar

[25] A. G. Howard, M. Zhu, B. Chen et al., "Mobilenets: efficient convolutional neural networks for mobile vision applications," 2017, https://arxiv.org/abs/1704.04861.View at: Google Scholar

[26] X. Zhang, X. Zhou, M. Lin, and J. Sun, "Shufflenet: an extremely efficient convolutional neural network for mobile devices," in Proceedings of the IEEE Conference on Computer Vision and Pattern Recognition, pp. 6848-6856, Salt Lake City, UT, USA, June 2018.View at: Publisher Site | Google Scholar

[27] G. Huang, Z. Liu, L. Van Der Maaten, and K. Q. Weinberger, "Densely connected convolutional networks," in Proceedings of the IEEE Conference on Computer Vision and Pattern Recognition, pp. 4700-4708, Honolulu, HI, USA, July 2017.View at: Publisher Site $\mid \underline{\text { Google Scholar }}$ 
[28] Li, L., Qin, L., Xu, Z., Yin, Y., Wang, X., Kong, B., ... and Cao, K. (2020). Artificial intelligence distinguishes COVID-19 from community-acquired pneumonia on chest CT. Radiology, 200905.

[29] Xu, X., Jiang, X., Ma, C., Du, P., Li, X., Lv, S., ... and Li, Y. (2020). Deep learning system to screen coronavirus disease 2019 pneumonia. arXiv preprint arXiv:2002.09334.

[30] Ghoshal, B., and Tucker, A. (2020). Estimating uncertainty and interpretability in deep learning for coronavirus (COVID-19) detection. arXiv preprint arXiv:2003.10769.

[31] Wang, S., Kang, B., Ma, J., Zeng, X., Xiao, M., Guo, J., ... and Xu, B. (2020). A deep learning algorithm using CT images to screen for coronavirus disease (COVID-19). DOI: https://doi.org/10.1101/2020.02.14.20023028.

[32] Bai, X., Fang, C., Zhou, Y., Bai, S., Liu, Z., Chen, Q., ... and Song, D. (2020). Predicting COVID-19 malignant progression with AI techniques. DOI: https://doi.org/10.1101/2020.03.20.20037325.

[33] Jin, C., Chen, W., Cao, Y., Xu, Z., Zhang, X., Deng, L., ... and Feng, J. (2020). Development and evaluation of an AI system for COVID19. DOI: https://doi.org/10.1101/2020.03.20.20039834.

[34] Jin, S., Wang, B., Xu, H., Luo, C., Wei, L., Zhao, W., ... and Sun, W. (2020). AI-assisted CT imaging analysis for COVID-19 screening: Building and deploying a medical AI system in four weeks. DOI: https://doi.org/10.1101/2020.03.19.20039354.

[35] Narin, A., Kaya, C., and Pamuk, Z. (2020). Automatic detection of coronavirus disease (COVID-19) using X-ray images and deep convolutional neural networks. arXiv preprint arXiv:2003.10849

[36] Wang, L., and Wong, A. (2020). COVID-Net: A tailored deep convolutional neural network design for the detection of COVID-19 cases from chest radiography images. arXiv preprint arXiv:2003.09871.

[37] Chowdhury, M. E., Rahman, T., Khandakar, A., Mazhar, R., Kadir, M. A., Mahbub, Z. B., ... and Reaz, M. B. I. (2020). Can AI help in screening viral and COVID-19 pneumonia?. arXiv preprint arXiv:2003.13145.

[38] Maghdid, H. S., Asaad, A. T., Ghafoor, K. Z., Sadiq, A. S., and Khan, M. K. (2020). Diagnosing COVID-19 pneumonia from X-ray and CT images using deep learning and transfer learning algorithms. arXiv preprint arXiv:2004.00038.

[39] D. Zhao, D. Zhu, J. Lu, Y. Luo, and G. Zhang, "Synthetic medical images using F\&BGAN for improved lung nodules classication by multi-scale VGG16," Symmetry, vol. 10, no. 10, p. 519, 2018.

[40] A. Beers, J. Brown, K. Chang, J. P. Campbell, S. Ostmo, M. F. Chiang, and J. Kalpathy-Cramer, "High-resolution medical image synthesis using progressively grown generative adversarial networks," 2018, arXiv:1805.03144. [Online]. Available: http://arxiv.org/abs/1805. 03144

[41] W. Dai, J. Doyle, X. Liang, H. Zhang, N. Dong, Y. Li, and E. P. Xing, "SCAN: Structure correcting adversarial network for chest Xrays organ segmentation," 2017, arXiv:1703.08770. [Online]. Available: https://arxiv.org/abs/1703.08770

[42] D. Nie, R. Trullo, J. Lian, C. Petitjean, S. Ruan, Q. Wang, and D. Shen, "Medical image synthesis with context-aware generative adversarial networks," in Proc. Int. Conf. Med. Image Comput. Comput.-Assist. Intervent., vol. 10435, 2017 , pp. 417425.

[43] Y. Xue, T. Xu, H. Zhang, L. R. Long, and X. Huang, "SegAN: Adversarial network with multi-scale 11 loss for medical image segmentation," Neuroinformatics, vol. 16, nos. 34, pp. 383392, Oct. 2018.

[44] T. Schlegl, P. Seeböck, S. M. Waldstein, U. Schmidt-Erfurth, and G. Langs, 'Unsupervised anomaly detection with generative adversarial networks to guide marker discovery," in Proc. IPMI, 2017, pp. 146157.

[45] Y. Wang, C. Dong, Y. Hu, C. Li, Q. Ren, X. Zhang, H. Shi, and M. Zhou, 'Temporal changes of CT ndings in 90 patients withCOVID19 pneumonia: A longitudinal study," Radiology, Mar. 2020, Art. no. 200843. [Online]. Available: http://10.1148/radiol.2020200843

[46] M.-Y. Ng, E. Y. Lee, J. Yang, F. Yang, X. Li, H. Wang, M.-M.-S. Lui, C. S.-Y. Lo, B. Leung, P.-L. Khong, C. K.-M. Hui, K.-Y. Yuen, and M. D. Kuo, "Imaging prole of the COVID-19 infection: Radiologic ndings and literature review," Radiol., Cardiothoracic Imag., vol. 2, no. 1, 2020, Art. no. e200034.

[47] C. Huang et al., "Clinical features of patients infected with 2019 novel coronavirus in Wuhan, China," Lancet, vol. 395, pp. 497506, Feb. 2020.

[48] L.Wang and A.Wong, “COVID-Net: A tailored deep convolutional neural network design for detection of COVID-19 cases from chest radiography images," 2020, arXiv:2003.09871. https://arxiv.org/abs/2003.09871

[49] L. Zhong, L. Mu, J. Li, J. Wang, Z. Yin, and D. Liu, “Early prediction of the 2019 novel coronavirus outbreak in the mainland China based on simple mathematical model," IEEE Access, vol. 8, pp. 5176151769, 2020.

[50] A. Imran, I. Posokhova, H. N. Qureshi, U. Masood, S. Riaz, K. Ali, C. N. John, M. Nabeel, and I. Hussain, “AI4COVID-19: AI enabled preliminary diagnosis for COVID-19 from cough samples via an app," 2020, arXiv:2004.01275. [Online]. Available: http://arxiv.org/abs/2004.01275

[51] IEEE Covid Chest X-Ray Dataset. Accessed: Mar. 7, 2020. [Online]. Available: https://github.com/ieee8023/covid-chestxray-dataset

[52] Covid19 Radiography Database. Accessed: Mar. 7, 2020. [Online]. Available: https://www.kaggle.com/tawsifurrahman/covid19radiography-database

[53] COVID-19 Chest X-Ray Dataset Initiative. Accessed: Mar. 7, 2020. [Online]. Available: https://github.com/agchung/Figure1COVIDchestxray-dataset

[54] D. P. Kingma and J. Ba, “Adam: A method for stochastic optimization," Dec. 22, 2014, arXiv:1412.6980. [Online]. Available: https://arxiv.org/abs/1412.6980

[55] A. Odena, C. Olah, and J. Shlens, "Conditional image synthesis with auxiliary classier GANs," 2016, arXiv:1610.09585. [Online]. Available: http://arxiv.org/abs/1610.09585

[56] F. Chollet. (2015). Keras: Deep Learning for Humans. [Online]. Available: https://github.com/fchollet/keras 


\section{A. Appendix:}

Fig. 1 - A flow-chart for diagnosis of COVID-19. As the sensitivity of RT-PCR is less so consecutive twice RT-PCR test is conducted for conformity.

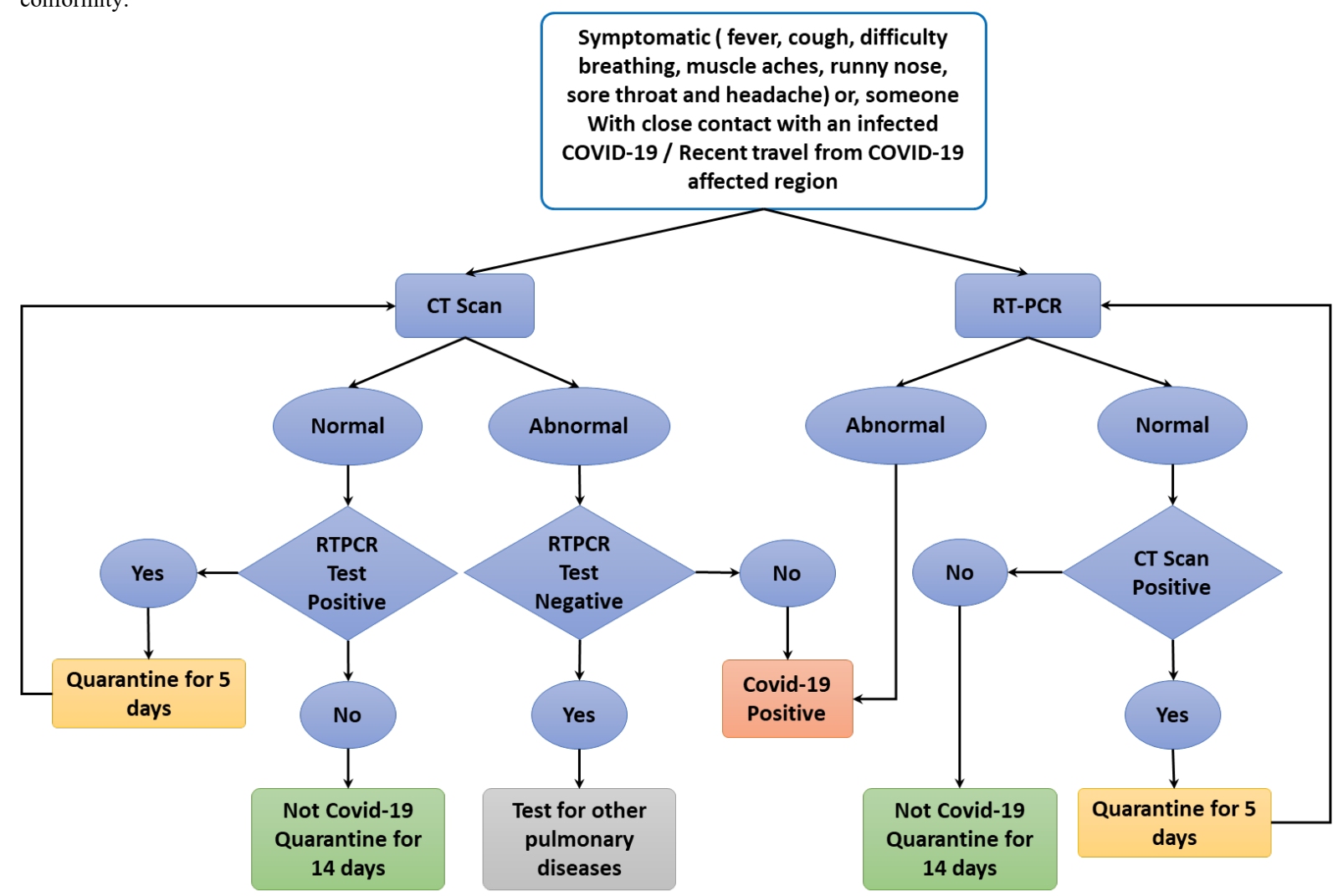


Fig. 2 CT image findings from the images.
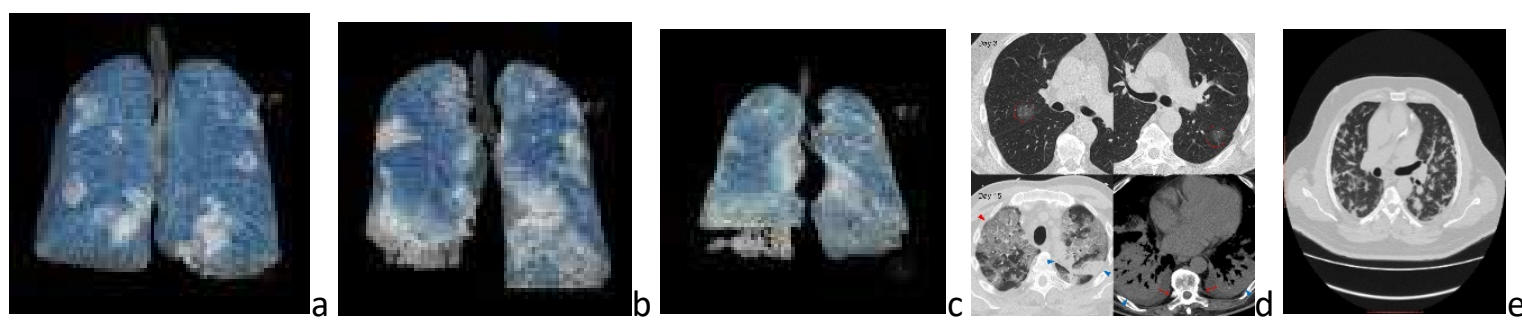

Fig 4- (a) CT demonstrates multilobar and bilateral ground-glass opacities with rounded morphology, mostly in the periphery of both lungs. There is no mediastinal hilar or axillary lymphadenopathy, nor pleural/pericardial effusion. This patient tested positive for coronavirus disease-19 and had typical CT findings of the disease. Thus, his diagnosis is COVID-19 pneumonia. (b) CT shows bilateral, multilobar large areas of ground-glass opacities with septal thickening and a crazy-paving pattern, associated with airspace consolidations. Lesions are mostly in the peripheral and posterior parts of the lungs. There are no pleural or pericardial effusion. This patient tested positive for SARS-CoV-2 and had typical CT findings of the disease. Thus, it was deemed to have COVID-19 pneumonia, (c) CT demonstrates multifocal and bilateral ground-glass opacities scattered in both lungs, predominantly at peripheral, and posterior regions. An associated overlying intralobular septal line is giving a crazy-paving pattern. No significant mediastinal lymphadenopathy. No pericardial or pleural effusion. This CT shows the typical CT findings of COVID-19 pneumonia, which was confirmed by a nasopharyngeal swab.(d) Findings for day 3:coronary sclerosis, mild aortic sclerosis, no significant mediastinal lymphadenopathy, two small areas of ground-glass opacity (GGO) in the posterior segment of the right upper lobe just above the fissures and in the upper segment of the left lower lobe, dilated intrahepatic and extrahepatic, bile ducts, Impression: Findings are suggestive of COVID-19 pneumonia. Biliary obstruction. (e) Ground-glass and consolidative opacities with minimal smooth interlobular septal thickenings noted in both lungs with peripheral as well as central distribution. No significant mediastinal/hilar lymphadenopathy noted. No pleural effusion noted.

Fig 3. Examples of chest CT images showing the segmented lungs image of patient with COVID-19.
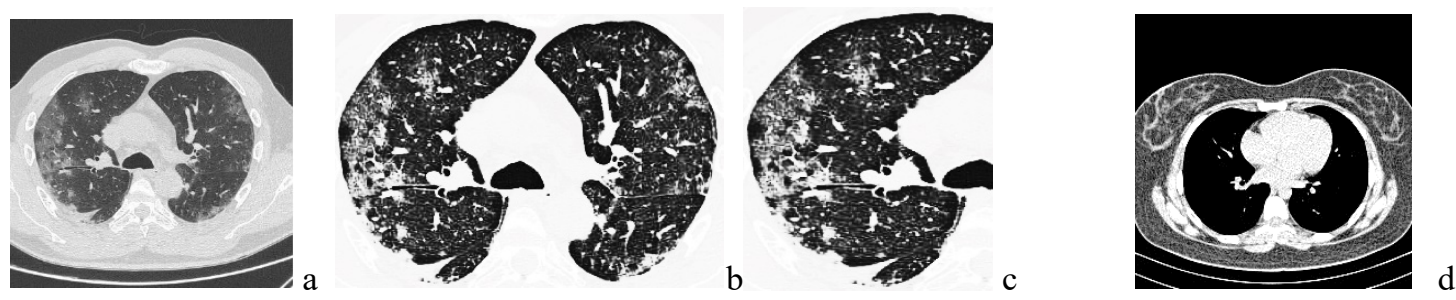

(a)The above mentioned 70-year-old was admitted with choledocholithiasis. COVID-19 contact. SARS-CoV-2 negative PCR on admission. Three days later cough and fever. Findings - day 3: coronary sclerosis, mild aortic sclerosis, no significant mediastinal lymphadenopathy, two small areas of ground-glass opacity (GGO) in the posterior segment of the, right upper lobe just above the fissures and in the upper segment of the left lower lobe, dilated intrahepatic and extrahepatic bile ducts. Impression: Findings are suggestive of COVID-19 pneumonia. Biliary obstruction. The last 2 images are manually segmented to aid radiologist for identification. (b) segmented lung images. (c) each lobe segmented for better understanding. (d) healthy lungs image.

\section{A.1. Tables}

Table 1 - Characteristics of patient's clinical information.

\begin{tabular}{|l|l|l|l|l|l|}
\hline Sex & Age & Clinical Symptoms & Exposure History & $\begin{array}{l}\text { Laboratory } \\
\text { Findings }\end{array}$ & $\begin{array}{c}\text { Radiological } \\
\text { Findings }\end{array}$ \\
\hline Men & $\begin{array}{l}50.55(\mathrm{avg}) \\
(25,85)\end{array}$ & $\begin{array}{l}\text { Fever } \\
\text { cough }\end{array}$ & 14 & 31 & 110 \\
\hline
\end{tabular}




\begin{tabular}{|c|c|c|c|c|c|}
\hline Female & $\begin{array}{l}60(\mathrm{avg}) \\
(30,70)\end{array}$ & $\begin{array}{l}\text { shortness of breath } \\
\text { dyspnea } \\
\text { sore throat, } \\
\text { dizziness, } \\
\text { lymphopenia. } \\
\text { myalgia } \\
\mathrm{SpO} 2 \text { of } 70 \%-89 \%\end{array}$ & $\begin{array}{l}\text { (Asymptomatic with } \\
\text { close contact } \\
\text { with an infected } \\
\text { COVID-19 } \\
\text { Recent travel from } \\
\text { COVID-19 } \\
\text { affected region) }\end{array}$ & $\begin{array}{l}\text { (Hypotension, } \\
\text { elevation of D- } \\
\text { Dimer. } \\
\text { pancreatectomy. } \\
\text { Renal failure } \\
\text { with } \\
\text { dysfunctional) }\end{array}$ & $\begin{array}{l}\text { (Classic, } \\
\text { Intermediate, } \\
\text { Un-identified at } \\
\text { first encounter) }\end{array}$ \\
\hline
\end{tabular}

Table 2 - Characteristics of patient's clinical information.

\begin{tabular}{|c|c|c|c|c|c|c|c|}
\hline $\begin{array}{l}\text { Serial } \\
\text { No. }\end{array}$ & Sex & Age & Cough & Fever & Other & Comorbidity & $\begin{array}{l}\text { Radiological Findings vs } \\
\text { RT-PCR Test }\end{array}$ \\
\hline 1 & & 45 & Yes & Yes & saturation of oxygen was $75 \%$ & No & Classic \\
\hline 2 & Male & 55 & No & 5 days & dyspnea & & Intermediate \\
\hline 3 & Male & 70 & No & 1 day & confusion with desaturation & & Classic \\
\hline 4 & Female & 60 & No & 5 days & dyspnea & & Intermediate \\
\hline 5 & Female & 30 & No & No & $\begin{array}{l}\text { Asymptomatic close contact } \\
\text { with an infected COVID-19 }\end{array}$ & & $\begin{array}{l}\text { First Swab confirmed } \\
\text { then radiological } \\
\text { images confirmed }\end{array}$ \\
\hline 6 & Male & 45 & 7 days & 7 days & $\begin{array}{l}\text { shortness of breath (dyspnea } \\
++)\end{array}$ & & Classic \\
\hline 7 & Male & 35 & 8 days & 8 days & dyspnea & & Intermediate \\
\hline 8 & Female & 30 & No & No & dyspnea & $\begin{array}{l}\text { Asthma, Cerebral } \\
\text { palsy }\end{array}$ & Intermediate \\
\hline 9 & Male & 45 & Yes & Yes & shortness of breath & & Intermediate \\
\hline 10 & Female & 70 & 15 days & 15 days & Sore throat, dizziness & $\begin{array}{l}\text { Hypotension, } \\
\text { desaturation, and } \\
\text { elevation of D- } \\
\text { Dimer. }\end{array}$ & Intermediate \\
\hline 11 & 75 & Male & No & No & $\begin{array}{lll}\begin{array}{l}\text { respiratory } \\
\text { dyspnea }\end{array} & \text { distress } & \text { and } \\
\end{array}$ & & Classic \\
\hline 12 & & Female & No & Yes & dyspnea & heart failure & Classic \\
\hline 13 & 60 & Male & 6 days & 6 days & & & $\begin{array}{l}\text { Negative First, then } \\
\text { confirmed swab test }\end{array}$ \\
\hline 14 & 70 & Male & Yes & Yes & $\begin{array}{l}\mathrm{SpO} 2 \quad \text { of } \\
\text { lymphopenia }\end{array}$ & & $\begin{array}{l}\text { Microbiologic test } \\
\text { was positive to } \\
\text { SARS-CoV-2 }\end{array}$ \\
\hline 15 & 55 & Male & Yes & Yes & $\begin{array}{l}\text { Lymphopenia, } \\
\text { dyspnea }\end{array}$ & & $\begin{array}{l}\text { After chest } \mathrm{x} \text {-ray, } \mathrm{PCR} \text { was } \\
\text { positive to SARS-CoV-2 } \\
\text { RNA. }\end{array}$ \\
\hline 16 & 70 & Female & No & 2 days & odynophagia & & Classic \\
\hline 17 & 50 & Male & 2 weeks & & $\begin{array}{l}\text { shortness of breath, } \\
\text { orthopnea, fever, myalgia, } \\
\text { and altered taste sensation for } \\
\text { last three days }\end{array}$ & $\begin{array}{l}\text { high LDH, ferritin, } \\
\text { CRP, and creatine } \\
\text { kinase (CK, CPK) } \\
\text { levels. WBCs, D- } \\
\text { dimer, CK-MB \& } \\
\text { troponin I were } \\
\text { normal }\end{array}$ & Intermediate \\
\hline 18 & 85 & Male & No & No & $\begin{array}{l}\text { ETOH excess. Lymphopenia } \\
\text { dyspnea }\end{array}$ & $\begin{array}{l}\text { Past history of } \\
\text { COPD. T2DM. } \\
\text { pancreatectomy. }\end{array}$ & $\begin{array}{l}\text { Chest } r \text { radiograph } \\
\text { suspicious of COVID-19, } \\
\text { which was later PCR } \\
\text { confirmed. }\end{array}$ \\
\hline
\end{tabular}




\begin{tabular}{|c|c|c|c|c|c|c|c|}
\hline 19 & 70 & & No & No & $\begin{array}{l}\text { Admitted with } \\
\text { choledocholithiasis. SARS- } \\
\text { CoV-2 negative PCR on } \\
\text { admission. Three days later } \\
\text { cough and fever. }\end{array}$ & & $\begin{array}{l}\text { A second real-time } \\
\text { reverse transcriptase- } \\
\text { polymerase chain } \\
\text { reaction (PCR) test of } \\
\text { the pharyngeal flora } \\
\text { just after the first CT } \\
\text { was positive for } \\
\text { SARS-CoV-2 virus } \\
\text { RNA. }\end{array}$ \\
\hline 20 & 70 & Female & 3 days & 3 days & & $\begin{array}{l}\text { vascath in-situ for } \\
\text { dialysis on a } \\
\text { background of } \\
\text { chronic end-stage } \\
\text { renal failure with } \\
\text { dysfunctional A-V } \\
\text { fistula. }\end{array}$ & $\begin{array}{l}\text { Nasopharyngeal swabs } \\
\text { returned positive for } \\
\text { COVID-19 on two serial } \\
\text { tests }\end{array}$ \\
\hline
\end{tabular}

